first place where I looked for the pus was in the temporosphenoidal lobe. The subsequent progress of the wound showed that I had over-estimated the danger of sepsis from contamination from the ear. It has been a constant source of regret to me that I was out of town on the Saturday evening when the symptoms supervened and therefore could not operate at once. It is also a source of regret to me that the case occurred during the holiday season, when all the physicians, except one, were away, and the message which I sent asking him to see the case never reached him; I was, therefore, left to my own unaided resources. The question which is of chief importance in these cases, and that on which I hope we shall have the opinion of some of the Fellows present is, What is the minimum of symptoms which justifies exploratory trephining?

One word also as to the position of the trephine opening, a point about which there has been a good deal of controversy. It seems to me that it is not possible to indicate one point which will suit al: cases. In this particular instance I selected the position indicated by Mr. Barker, because, from the entire absence of symptoms localising the abscess, I concluded that it was most probably situated about the middle of the temporo-sphenoidal lobe.

The cause of the rigors is not by any means clear. I naturally at first concluded that pyæmia had set in, but the further progress of the case negatived this view. There was no evidence at any time of thrombosis of the lateral sinus. The diagnosis was made still more perplexing by the swelling of the scalp which followed the application of the alembroth gauze, and to some who saw the case this swelling seemed to indicate a suppurative periostitis or osteomyelitis extending from the trephine hole. This was a view which I could not, however, accept, for the swelling of the scalp was coextensive with the dressing from the first, that is, occupied about half the scalp, while I should have expected an osteomyelitis to be limited to the temporal bone; and further the dermatitis which was evidently present exactly resembled what one had previously seen from the action of sal alembroth on the skin in other parts of the body.

The meaning of the temporary paralysis is also obscure. At first it seemed to point to a purulent leptomeningitis, but the ultimate recovery of the patient seems to preclude that view, and I presume it must have been due to some temporary congestion of the cortex of the brain, probably in connection with the formation of the second collection of pus, or possibly the result of probing the brain with the riew of finding pus.

Lastly we have to note the formation of a second collection of pus, which might either have been reaccumulation in some diverticulum of the original abscess, which was not perfectly drained, or an entirely fresh formation in the neighbourhood of the original collection. I am inclined to take the latter view, because the second abscess was situated distinctly further back and higher up than the first, and it seems to me that the rigors, relapse, and other symptoms which afterwards developed were mainly connected with the formation of this second abscess. In connection with the opening of this second abscess there is one practical point to which I think it is worth while to call attention. 1 searched the situation of the abscess a few days before it was opened with a hollow needle and failed to find pus; a few days later I introduced sinus forceps and on expanding the blades, pus at once welled out. Now I have found on several occasions, and also in this case, that when a hollow needle is passed through healthy brain tissue it at once becomes filled with a plug of brain material, and even though it enters a collection of fluid, unless that fluid is under high tension, this plug is not forced out, and the fluid may remain undetected. On the other hand by using fine sinus forceps much less injury is done to the brain and, by expanding the blades from time to time, if fluid is reached it will certainly appear at the surface. I venture therefore to recommend the use of long sinus forceps in place of hollow needles when one wishes to search for collections of fluld in the brain.

Dr. Alexander M a carkgor, Assistant Physician to the Aberdeen Hospital for Sick Children, has commenced the publication of a series of demonstrations in clinical medicine. They are intended for students attending his clinique, and are given to them gratis. Their issue is promised at regular intervals. The first is a demonstration of a case of hemiplegia, with illustrated diagrams and clinical commentary. There appears to be a general desire among students that teachers might publish more fully in this

\section{REPORT OF AN EPIDEMIC OF INFLUENZA} (140 CASES) OCCURRING AT THE ROYAL ASYLUM, MORNINGSIDE, EDINBURGH.

By GEORGE M. ROBERTSON, M.B., AND

FRANK A. ELKINS, M.B., Assistant Physicians.

Wr here record, with the permission of Dr. Clouston, our experiences of a severe outbreak of influenza, occurring in this ssylum. As assistant-physicians in an asylum we have had the advantage of seeing our cases from the very commencement of the attacks, and of following them to the end. Our observations have also been made on a population of both sexes, and of known numbers.

\begin{tabular}{|c|c|c|c|c|c|c|}
\hline Patients & …....... & $\begin{array}{r}\text { Male. } \\
407 \\
71\end{array}$ & …....... & $\begin{array}{c}\text { Female. } \\
410 \\
104\end{array}$ & …......... & $\begin{array}{c}\text { Total } \\
817 \\
175\end{array}$ \\
\hline ot & ......... & 478 & ........... & $\overline{514}$ & ........... & $\overrightarrow{992}$ \\
\hline
\end{tabular}

Commencement of Outbreak.-During the second and third weeks of December, scattered cases of illness, which we did not at the time differentiate from ordinary colds, occurred; but during the fourth week of the month we became certain that the epidemic was amongst us, by the daily invasion, by the numbers attacked, and the nature of the symptoms. By the end of the first week of January there had been about fifty cases, and during the second week about sixty more were added to the list. Since then the cases have been fewer in number, and at the present time (January 24th) we have only two or three cases, excluding those still suffering from sequele.

General Summary of Symptoms.-The symptoms have been extremely various and numerous, but we have noted the following as the principal ones. The symptoms which almost always occurred were frontal headache and bodily pains. The headache was sometimes very severe, and was accompanied by giddiness. It almost invariably passed away in twenty-four hours. The pain most complained of was in the lumbar region, resembling lumbago, but this was usually accompanied by neuralgic or muscular pains in many other parts, especially in the legs. These pains were much more persistent than the headache, and occasionally remained during convalescence. A slight respiratory catarrh usually accompanied these symptoms. There was a dry cough, with a tough scanty sputum, and there was coryza with suffusion of the eyes. The "running" from the eyes and nose, though often present, was not very prominent in our cases, nor was sneezing a marked symptom. The temperature was an important guide, and at the beginning of well marked cases was almost always above $100^{\circ}$. The attack was marked by suddenness of onset and a few hours before the headache and rise of temperature occurred the patient appeared and felt in his usual bealth. A slight rigor or a feeling of chilliness usually ushered in the attack. After a duration of about three days, during which time the symptoms gradually disappeared, the temperature fell to the normal. The convalescence was slow, and was marked in almost all cases by extreme weakness.

Having given an outline of the disease, we will now describe in detail the various symptoms.

\section{Analysis of Particular Symptovs.}

Cerebral Symptoms.-The frontal headache has already been mentioned as being the most constant, and it was also the symptom most complained of. Along with this there was always a disagreeable feeling described as giddiness, lightheadedness, or swimming in the head. The highest nervous functions were affected. There was generally considerable mental depression, and a complete "giving in" to the disease without desire or ability to resist, the patient at once taking to his bed. There was also amongst the sane a want of attention and concentration of thought, so that reading and discharge of business was rendered impossible. This often persisted for a day or two after recovery. The sleep, especially during the first night of the disorder, was disturbed by disagreeable dreams, and in four of our cases delirium occurred. Most cases complained of sleeplessness, and in four cases there was persistent insomnia neces- 
sitating the use of hypnotics. In cases of delusional insanity, especially of the suspicious variety, the mental symptoms were aggravated.

Neuro-muscular Symptoms.-Pain in the lumbar region has been mentioned amongst the constant symptoms, and was nearly as distressing to the patient as the frontal headache. Pains of a similar character might exist in any region of the body, the patients often saying that they felt as if they "had been beaten" all over. Next in frequency to the back, the calves of the legs and the thighs were most affected by the pains, and in one case (a female nurse) this symptom was so severe as to induce a hysterical condition. Usually there was no increase of pain on pressure. There was often a deep-seated aching of the bones of the extremities, and only very seldom were the pains localised at the joints. The pains were often of a purely neuralgic character, rarely localised to one spot, and generally of a darting and shooting character in the limbs. Intercostal neuralgia was complained of, and also aching pains in the eyeballs, increased on pressure. The feeling of water being poured down the back, as well as flushings of heat, were frequently noted, and these symptoms often persisted during convalescence. During the attack there was a feeling of muscular weakness, in many cases amounting to prostration, and in six cases there was fainting. During convalescence there was almost invariably great weakness complained of for two or three days, so that fainting has also occurred at this period. This muscular weakness we consider a most important symptom of the ailment. It was alluded to without suggestion by almost all the patients, and by statistics we found it to be the most frequent symptom. After the attack the handwriting was shaky and deteriorated and the gait occasionally unsteady, even when marked weakness had dis appeared. The convalescing patient was quite unfit for either mental or bodily work. Marked rigors were rare, but in the large majority of cases slight ones occurred. Patients seldom referred to them unless directly questioned on the subject.

Alimentary Symptoms. - The appetite was always impaired, and in many cases there was loathing of food and nausea. Vomiting was frequent as an initial symptom, and in some cases continued for a day or two, and caused great exhaustion. Constipation often occurred, while in a smaller number of cases there was diarrhœa, in two cases with blood in the stools. The diarrhœe was hardly ever an initial symptom; it was sometimes preceded by constipation, and was readily set up by laxatives. Flatulence was a common symptom, and was accompanied by severe "colicky" pains. In some cases there was pain and tenderness on pressure in the region of the liver. The tongue was very slightly furred in the majority of cases, but in those with severe abdominal symptoms there was a thick creamy fur.

Respiratory Symptoms.-The symptom of cold in the head was frequently, but certainly not invariably, present. If there was a discharge it was slight, watery, and only of a few hours' duration, although it might recur. Sneezing was rare. The conjunctivæ were suffused in many cases, and there was often slight lachrymation. In those cases in which the above symptoms were well marked, there was swelling of the nose, accompanied by a redness and swollen appearance of the eyelids. Epistaxis occurred in two cases. Slight transitory hoarseness and sore throat were often complained of, and on examination only congestion of the pharynx and tonsils was observed. Over threefourths of the cases complained of a dry cough; it was occasionally troublesome, and generally persisted well on in convalescence. The sputum was scanty, tough, and difficult to get up, and was not present during the first day or two. Pain in the chest, as in bronchitis, was sometimes complained of. Bxamination of these cases always revealed sparse dry râles, and in some cases moist râles, at the bases. Several of these cases passed into acute bronchitis, pleurisy, and pneumonia, and all the fatal cases were due to chest complications.

Circulatory Symptoms.-During the fever the pulse was of course quickened, and during convalescence it was soft, small, and weak, but we observed nothing else of special value regarding it. As regards the condition of the heart, a most serious and not infrequent complication was dry pericarditis. The skin was usually congested, and over the face and trunk, more especially, there was a reddish flush when the temperature was high. The face had occasionally a dusky congested look. No decided eruption was noticed, but in three cases there was herpes labialis. The patients complained greatly of waves of heat and cold passing over the body, accompanied by periodic copious perspirations. This latter symptom was much more marked than in ordinary febrile states, and indeed persisted after the temperature reached the normal.

Urinary Symptoms.-Observations on the urine under the circumstances were necessarily imperfect, but in two cases there was acute catarrhal nephritis.

The Temperature was an important guide, and was always above $100^{\circ}$ in a typical case. When there were no complications it was always higher the first evening than subsequently, and throughout the attack the evening was almost invariably higher than the morning temperature. Unless there were complications we found the temperature fell gradually, with evening remissions, till it reached the normal. In four cases without any apparent complication it was above $104^{\circ}$ at first ; in fifteen cases, or about 10 per cent., it was above $103^{\circ}$, but, in the vast majority of cases, the temperature at its highest was between $100^{\circ}$ and $102^{\circ}$.

The duration of typical cases, judging by the temperature, was from two to five days, and on an average about three and a half days. The symptoms are almost invariably much abated after the first day, although, like the temperature, there is an aggravation in the evenings. We must here mention that we have had somewhat numerous cases, showing some of the symptoms of influenza in a mild form, such as coryza, headaches, or pains and aches. In these cases the temperature might be a few points above normal, but the symptoms passed off in a day or so, and the patients did not require to go to bed. These cases are not included in our statistics.

During convalescence the patients felt well whilst in bed, and were very anxious to rise, but on getting up discovered that they were exceedingly weak and giddy. When they attempted to go about and do work they found themselves by evening completely exhausted. They still remained subject to shiverings, flushings, and perspirations, and there was a liability to relapse.

Sequele.-The rheumatic pains in some parts of the body might last for some days or a week. Several cases complained of sore throat, and the majority had a slight cough for a week after the attack. Anæmia to a greater or lesser extent was always present, and although all lost weight during the illness, some, especially those with abdominal symptoms, became much reduced and very weak. The appetite remained very poor.

Relapses.-So far we have had 13 relapses, 9 being males, and 4 females. In other words, of the 140 attacked, 9.2 per cent. have relapsed (12.3 per cent. males, and 5.9 per cent. females). As a general rule the second attack was more serious than the first, and headache and fever always recurred. The majority of cases had a severe pulmonary attack (bronchitis or pleurisy), and in nearly a half diarrhœa occurred. In all relapsed cases there was some distinct exposure to cold air, usually by going out of doors too soon, and in no case did a relapse occur so long as the patient was in bed or strictly confined to a heated room. The period elapsing between the exposure and the symptoms of the second attack was very short; in 4 of the 13 cases the relapse occurred the day after going out of doors, and in 3 others it occurred within 24 hours of leaving the heated sick room for the purpose of going to the watercloset, where ventilation is active. Our opinion of the period of incubation of the first attack is that it is very brief, and that its duration is within 24 hours, though we have few definite observations on this point.

Complications.-The most frequent complication, and one which occurred in all the fatal cases, was a species of pneumonia. It occurred in 14 cases, 13 males and 1 female, of which 10 males have died, and one more male is likely to succumb. It attacks both bases as a rule, and is accompanied by a high temperature, always above $104^{\circ}$, in three cases $106^{\circ}$, and in one case $108^{\circ}$ previous to death. It must be remembered, however, that some of these were cases of general paralysis of the insane, in which the temperature might rise from nervous causes. The pneumonia was not of the pure croupous variety; bronchitis always preceded it, and it seemed to be rather a spread of the inflammatory process to the terminal tubules of the bronchi, and finally to the alveoli. The condensation was gradual, and the respiratory accompaniments were always crepitations of the median coarse variety, with a few dry râles. The sputum, though fibrinous, was frothy, and, though occasionally streaked with blood, was never rusty-coloured. Bronchitis, though not so serious a complication, was a more common one, and frequently required active treatment. Pleurisy, always the dry form, occurred in several cases, sometimes singly, but generally accompanying the pneumonia. Pericarditis was discovered in 5 of the 10 fatal cases, and in one other case. In 2 there has been dcute catarrhal nephritis, and in 2 others subacute rheumatism. 
Deaths. - There have been 10 deaths, all male patients. The main cause of death in all these cases has been the pneumonic condition, assisted greatly, however, by the very weak general health of the patients attacked. All of the 10 cases were insane patients, and of these 6 were suffering from general paralysis and were in the third stage, 3 were over 70 years of age, and the other was an alcoholic case with very serious organic disease of the heart. It is obvious that no reliable conclusions can be drawn from these numbers which would be of use in general practice, but we give the percentage of deaths to the affected: 7.1 per cent. of the total number affected died; 13.6 per cent. of the males attacked died; and 19.6 per cent. of the insane males attacked died. No female patients, nor any official, male or female, died.

We here append a table of the symptoms in 70 unselected cases (38 males, 32 females), in which accurate observations were made, with the percentage of their frequency :

Great weakness after attack ...

Frontal headache $\quad \ldots$.

$\begin{array}{lllll} & \ldots & \ldots & \ldots & \\ \text { Loss of appetite } & \ldots & \ldots & \ldots & 81.4\end{array}$

$\begin{array}{llll} & \ldots & \ldots & 78.1\end{array}$

Coryza

Slight bronchitis

Suffusion of eyes

Rigor...

Nausea

$\cdots$

... 77.1

71.4

Unpleasant dreams, "wandering"

Flatulence

Vomiting

Constipation ...

Diarrhoea

$\cdots \cdot \quad \cdots$

... $\quad 58.6$

$\ldots 386$

... 37.1

$\ldots 25.7$

Having described the symptoms of the disease. we will now record *ome general facts. Out of a population of 992 persons, 140 were attacked, a percentage of 14.1, and of these 73 were males and 67 were females. Compared with thetotal number of males and females in the asylum, the percentages are 15.2 per cent. males and 13 per cent. females. It is thus seen that the males were slightly more subject to the disease than the females. The type of the disease was more severe among the males than the females, and this observation is strengthened by the following facts. Out of 13 relapses, 9 were males; out of 14 cases complicated with pneumonia, 13 were males; and of the 10 deaths, all were males. We suggest that this preference for the male is greatly due to the fact that the men are more in the open air, where we believe the disease is contracted. As a fact supporting this statement we find that the officials, amongst whom, moreover, the standard of health is high, were attacked in far greater proportion than the patients, the former of course having greater facilities of going about outside. Of the 175 officials 28.4 per cent. have suffered ( 30.9 males and 25.9 females), whilst of the 817 patients only 11.1 have been attacked (12.5 males and 9.7 females). The first persons attacked, moreover, were those who were most in the open air, the majority of them being officials, and of the patients attacked the majority were those who were much outside. Of the percentages attacked in the different wards the largest numbers on the male and female sides of the asylum were in the convalescent wards, where the "open door system" is adopted and the patients are at liberty to walk in the grounds. It has already been stated that relapses only occurred after exposure to outside air.

Contagion. - We can express no very definite opinion as regards the contagiousness of the disease; if however contagiousness exists, it only does so to a very slight degree. As pointing to contagion, we may state that it attacked four officials in the female hospital one after the other (three sleeping in one room), b 3 fore the disease had appeared elsewhere in the house. On the other hand, we have to state that excepting the female hospital, the disease appeared practically simultaneously in every ward of the house, and it did not tend to spread more in one ward than another, to any remarkable extent, as the following table shows. The following percentages being derived from small numbers (45 being the average in a ward), considerable latitude must be given for fallacies due to accidental circumstances.

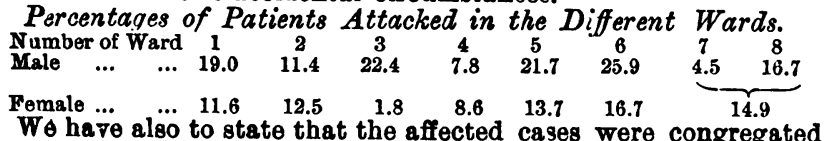

together and isolated as much as possible from the healthy patients, and on the male side, of the seren officials devoted to the sick, only one took the disease. The days during which most patients were attacked were the 2 nd to the 5th of January.

Our opinion of the disease is that it is certainly not a common cold, and we believe it to be a specific fever, having a definite course and duration, and affecting the whole organism. The nervous, muscular, respiratory, and alimentary systems are all affected, and, as in other fevers, this disease always leaves its victims debilitated. It is thus distinct from ordinary respiratory catarrhs; and, indeed, in 22.9 per cent. of the cases there was no catarrh of the nasal or bronchial membranes.

In no case $w$ is ther $\theta$ only a single system affected; we have found that the neuro-muscular system, under which we include headache, neuralgic and muscular pains, was affected in 97.1 per cent. of the cases; that the respiratory system, under which we include symptoms referred to the conjunctival, nasal, and bronchial membranes, was affected in 77.1 per cent. of the cases, and that the alimentary system, under which we include only vomiting or diarrhœa, was affected in 55.7 per cent. of the cases. It is thus seen that any sharp division of this disease into the neuro-muscular, respiratory, and alimentary varieties is artificial and by no means accurate At the same time, it is convenient from the clinical point of view to describe the varieties of the disease by the system which is most prominently involved.

Treatment. - When once it was evident that the disease was epidemic we reserved separate wards for its treatment, and whenever an individual was attacked, he or she was removed to these wards and kept in bed. The diet was light, mainly consisting of milk, many cases being able to take only milk and potash water. As regards the medicinal traatment, none of the drugs which we used affected in any noticeable degree the symptoms or course of the disease. For the high temperature we used antipyretics, and of these we preferred to use antipyrin in 10-grain doses in those cases in which headache was the most prominent symptom. When pain in the back or other rheumatic pains were greatly complained of we used salicylate of soda in 10-grain doses; we found however that it increased the nausea, and it was also objectionable from its reputed depressing effects. Quinine however in 10-grain doses was more used than either of the above remedies. For the pain in the back, if severe, we applied a mustard leaf, with relief in almost all cases. For the cure of constipation we adopted the use of soap and water enemata, having discovered by experience, that diarrhœa was readily set up by the mildest laxatives. For the bronchitis accompanied by tough sputum we prescribed: B Sp. ammon. aromat. $\xi j$; liq. ammon. acetat. ad $\xi \mathbf{j}$. Sig. A tablespoonful in water every three or four hours. For insomnia, sulphonal $30 \mathrm{grs}$, or paraldehyde, was prescribed, and the latter made up as follows: $\mathrm{R}$ Paraldehyde $3 j$ to $31 j$; tr. quillaim $3 \mathrm{ss}$; aq. cinnamomi ad $\xi i j$. Sig. The draught to be taken in water. During convalescence we prescribed E iston's or Parrish's syrup, quinine tonics, maltine, claret, port, stout, and bitter ale, according to circumstances, at the same time increasing the dietary. The complications were treated according to the ordinary methods. In conclusion we would strongly impress upon all attacked the urgent necessity of going to bed at once, and of staying there for three days after the temperature is normal.

We would here point out that our population is entirely an adult one, with many aged people, and as the general health is decidedly below the average, an epidemic such as this is a much more serious matter to us than to the general public.

EPPING RURAL (Population, 21,725).-Prevalence of Whoopingcough: Absence of Typhoid and Diphtheria in Epidemic Form. Reporting on the health of this district during 1888, Dr. Trevor Fowler states that a material increase in the zymotic death-rate was almost entirely caused by whooping-cough, the record of deaths from this cause being the highest of the past ten years. No less than 20 ont of 43 deaths attributed to zymotic diseases were from whooping-cough, and as death in these cases was almost invariably the result of exposure or other bad management, it is evident that the power to arrest such high mortality rests more with the people themselves than with the sanitary authority. Measles and scarlatina were also somewhat prevalent, but the character of the outbreaks was mild, and there were not many deaths. The district has been conspicuously free from typhoid and other allied fevers for the past five years, which is a very encouraging fact, and there has also been a total absence of diphtheria in epidemic form during both 1887 and 1888. The general death-rate was 15.5 per 1,000 , and the zymotic rate 1.8 . 